\title{
Deep tissue multiphoton microscopy based on advanced femtosecond fiber sources
}

\author{
Shih-Hsuan Chia ${ }^{1,2}$, Hsiang-Yu Chung ${ }^{2}$, Wei Liu ${ }^{2}$, Franz X. Kärtner ${ }^{2,3}$, and Guoqing Chang, \\ ${ }^{I}$ Institute of Biophotonics, National Yang-Ming University, 112 Taipei, Taiwan (ROC) \\ ${ }^{2}$ Center for Free-Electron Laser Science, DESY, and Physics Department, University of Hamburg, 22761 \\ Hamburg, Germany \\ ${ }^{3}$ The Hamburg Center for Ultrafast Imaging, 22761 Hamburg, Germany \\ ${ }^{4}$ Beijing National Laboratory for Condensed Matter Physics, Institute of Physics, Chinese Academy of Sciences, \\ Beijing 100190, China \\ Tel: +88622826 7249, e-mail: shchia@ym.edu.tw
}

\begin{abstract}
The use of multiphoton microscopy has proven its potential on deep tissue virtual biopsy for early diagnosis and neuronal processes imaging. However, the capability of multiphoton microscopy strongly depends on the availability of easily operated femtosecond laser sources. By precise management of fiber-optic nonlinearity, we have demonstrated the way to generate nearly transform-limited femtosecond sources with the tunable wavelength range of $0.9 \mu \mathrm{m}-1.2 \mu \mathrm{m}$, covering both the GFP two-photon-excitation band and transparency window of common bio-tissues. The demonstrated approach is fully energy scalable to sub-100nJ level of pulse energy with the spectral tunable range down to $1.31 \mu \mathrm{m}$, which paves the way to capture deep-tissue imaging contrast from third harmonic generation and three-photon-excited GFP fluorescence. We applied such an energetic source to a proof-ofprinciple study of ex vivo human skin based on harmonics (i.e., second-harmonic generation and third-harmonic generation) imaging. This new type of fiber-format energetic ultrafast source will be performed as a robust solution for multiphoton microscopy applications, as well as studying the damage assessments from thermal heating and multiphoton absorption during the imaging process.
\end{abstract}

Keywords: Multiphoton microscopy, femtosecond fiber laser, nonlinear fiber optics.

\section{INTRODUCTION}

Advanced microscopic technology to obtain deep-tissue images with cellular/subcellular information are desired for many biomedical research fields [1]-[4]. For example, the development of virtual biopsy technique providing pathohistological information paves a way to improve quality of life in terms of both definitive diagnosis in the early stage and treatment assessments [2, 3]. Nonlinear multi-photon signal contrast [1]-[4], light sheet technique [5], and confocal method [6] have been widely applied to visualize the sample tomography with a possibly submicron optical resolution. Compared with other techniques, the strengths of nonlinear light microscopy on both the minimal constraint on the specimen and potentially deeper penetration depth are of great importance to increase the feasibility of in vivo animal/clinical observation.

The capability of nonlinear microscopy depends on the advancement of femtosecond sources: the excitation wavelength of the femtosecond pulses should be optimized to selectively excite exogenous/endogenous chromophore, or to mitigate tissue attenuation (i.e., scattering and absorption), allowing deeper imaging penetration. The driving wavelengths of nonlinear light imaging (two-photon fluorescence and second/third harmonic generation) are typically located in the near infrared regime with the pulse energy of $\sim 1 \mathrm{~nJ}[1-3]$. Much higher pulse energy (sub-100nJ) are even required for three photon fluorescence excitation, capable of $>1 \mathrm{~mm}$ imaging depth within an intact mouse brain [4]. Moreover, development of robust hands-off laser tool is crucial for applying nonlinear light microscope outside research environments. For the last two decades, femtosecond solid-state bulk lasers (e.g., Ti:sapphire lasers) have become the standard driving sources. However, such lasers demand precise cavity-alignment and environmental control (i.e., water cooling, temperature and humidity feedback loop, vibration isolation etc.), which limits the use of nonlinear light microscopy to specialized laboratories.

Femtosecond fiber lasers can be constructed from all polarization-maintaining fibers without any free-space elements $[7,8]$, undoubtedly offering robust solutions for deploying nonlinear microscopic systems in rugged environments especially for in vivo imaging and clinical applications. Unfortunately, the most mature femtosecond fiber lasers developed to date are Yb-fiber lasers in 1020-1060 nm and Er-fiber lasers in 1520-1570 nm. The demonstrations of such fiber-sources covering the typical multiphoton excitation band are challenging, requiring precise nonlinear frequency conversion from the gain region of Yb-fiber lasers (1030 nm) or Er-fiber lasers (1550 $\mathrm{nm}$ ) while maintaining sustainable pulse energies.

Soliton self-frequency shift (SSFS) inside an optical fiber has been applied to generate sub-100nJ pulse energy for three-photon fluorescence microscopy at around $1675 \mathrm{~nm}$ [4]: Output pulses from an ultrafast Er-fiber laser can be continuously wavelength red-shifted based on stimulated Raman scattering. SSFS requires negative groupvelocity dispersion (GVD) and can be realized in standard single-mode fibers and large-mode-area (LMA) 
photonic crystal fibers (PCFs) if the center wavelength of input pulses is larger than $\sim 1300 \mathrm{~nm}$ - the typical zerodispersion wavelength (ZDW) of the fundamental mode. Despite the successful wavelength-shift to $\sim 1700 \mathrm{~nm}$, energetic fiber-based femtosecond sources operating below $1350 \mathrm{~nm}$ remain out of reach. This wavelength window is particularly crucial for multiphoton imaging because the generated two-/three-photon signals (from the most commonly used fluorophores or from optical harmonics) are located at the visible wavelength range corresponding to optimal detection efficiency. In order to achieve SSFS for the excitation wavelengths below $1300 \mathrm{~nm}$, photonic crystal fibers (PCFs) with a small $(<3 \mu \mathrm{m})$ mode-field diameter (MFD) are usually employed to shift the ZDW below $1030 \mathrm{~nm}$ [9]. The associated strong nonlinearity in these PCFs limits the resulting wavelength-converted pulses to $<1 \mathrm{~nJ}$ in pulse energy. Dispersion in higher order-mode fibers can be employed to achieve SSFS with $>1$ $\mathrm{nJ}$ pulse energies from an Yb-fiber laser. However broadband mode-converting back to the fundamental modea necessary beam profile for most MPM systems - is challenging [10].

Despite of using SSFS, the method employs Kerr fiber-optic nonlinearities (dominated by self-phase modulation, SPM) can be a great candidate to broaden an input optical spectrum with much more design freedom [11,12]. The broadened spectrum can be well-controlled by selecting the fibers, changing the fiber length, and varying the input pulse energy. In addition, the broadened continuum spectrum enabled by SPM is possibly compressible without incoherent light generation from nonlinear light conversion. As a result, one can select the leftmost or rightmost spectral lobes using optical bandpass filters as a tunable excitation source by varying the characteristics of nonlinear fiber or input pulse energy. Without external compression, the filtered spectral lobes correspond to $\sim 100$ fs (nearly transform-limited) pulses, tunable from $825 \mathrm{~nm}$ to $1210 \mathrm{~nm}$ with $>1 \mathrm{~nJ}$ pulse energy [11]. For high pulse energy application such as third harmonic generation microscopy, we demonstrate the energy scalability of these SPM-enabled ultrafast fiber sources: up to $20 \mathrm{~nJ}$ pulses (<100 fs, 55-MHz repetition rate) tunable from 1030-1215 $\mathrm{nm}$ are obtained [12]. These key parameters represent a milestone in fiber laser technology aiming for the application of multiphoton microscopy, and the capability of two-/three-photon imaging is demonstrated by performing harmonic virtual biopsy of ex vivo human skin.

\section{FEMTOSECOND NONLINEAR FIBER SOURCES}

We started from a home-built Yb-fiber oscillator produces 55-MHz ultrashort pulses centered at $1033 \mathrm{~nm}$ with 70$\mathrm{mW}$ average power. Figure 1 depicts the schematic of our experimental setup. These pulses from the laser oscillator were first stretched to $\sim 20 \mathrm{ps}$, and then amplified to $10-\mathrm{W}$ average power by two amplifiers. After an optical isolator, a grating-pair compressor de-chirped the amplified pulses to $190 \mathrm{fs}$ in duration with 8-W average power. The temporal pulse shape after the laser system is carefully optimized by the dispersion compensation from the diffraction-grating pair and the length selection of the gain fiber and the stretcher fiber. The compressed pulses were coupled into an optical fiber for SPM-enabled spectral broadening followed by optical bandpass filters to select the rightmost/leftmost spectral lobe. A half-wave plate and a polarization beam splitter were used to adjust the power coupled into the nonlinear fiber. Finally, as a proof-of-principle, we employed the filtered femtosecond source to drive a video-rate laser scanning MPM microscope.

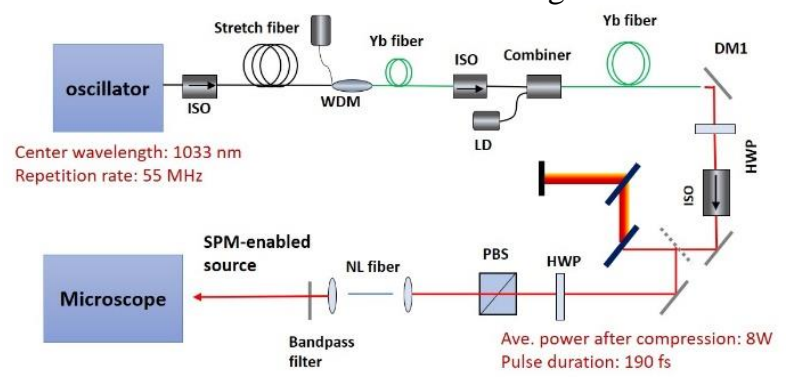

Figure 1. Experimental setup. ISO: isolator, WDM: wavelength division multiplexer, LD: laser diode, DM: dichroic mirror, HWP: half-wave plate, PBS: polarization beam splitter, NL fiber: nonlinear fiber

To demonstrate a widely wavelength-tunable ultrafast source with $>1 \mathrm{~nJ}$ pulse energy, one may use a short piece of nonlinear fiber (e.g., PCFs) with the ZDW around the laser center wavelength. For example, we varied the average power coupled into 20mm PCF NL-1050-ZERO-2 with the ZDW at $1050 \mathrm{~nm}$ and the MFD of $2.2 \mu \mathrm{m}$, and we used a series of optical bandpass filters to select the leftmost or the rightmost spectral lobes. The filtered spectra (Figure 2(a)) can be widely-tuned from $825 \mathrm{~nm}$ to $1210 \mathrm{~nm}$ with $>1 \mathrm{~nJ}$ pulse energies at a repetition rate of $55 \mathrm{MHz}$ [11]. Such spectral coverage and excitation pulse energy will be desired to replace the expensive Ti:sapphire laser system along with an optical parametric oscillator for two-photon fluorescence microscopy.

The output power can be fully scalable by using larger core-diameter fibers [1]. Fiber with a larger mode field diameter allows to handle higher coupled power. We varied the coupled average power into 70-mm fiber LMA-8. At 4-W coupled power in the fiber with the MFD of $7.5 \mu \mathrm{m}$, the output broadened spectrum spans from $850 \mathrm{~nm}$ to $1250 \mathrm{~nm}$, and the filtered rightmost spectral lobe red-shifts from $1050 \mathrm{~nm}$ to $1215 \mathrm{~nm}$ as shown in Figure 2(b). Another interesting feature is that the broadened spectra exhibit clear distinct spectral lobes around the 1100-1200 nm wavelength range, resulting from the low fiber dispersion in this spectral range. Fiber LMA-8 has the ZDW at 
$1160 \mathrm{~nm}$ and exhibits relatively small GVD in the wavelength range of 1100-1200 nm. Therefore, almost pure SPM-dominated continuum generation leads to the characteristic spectral lobes.
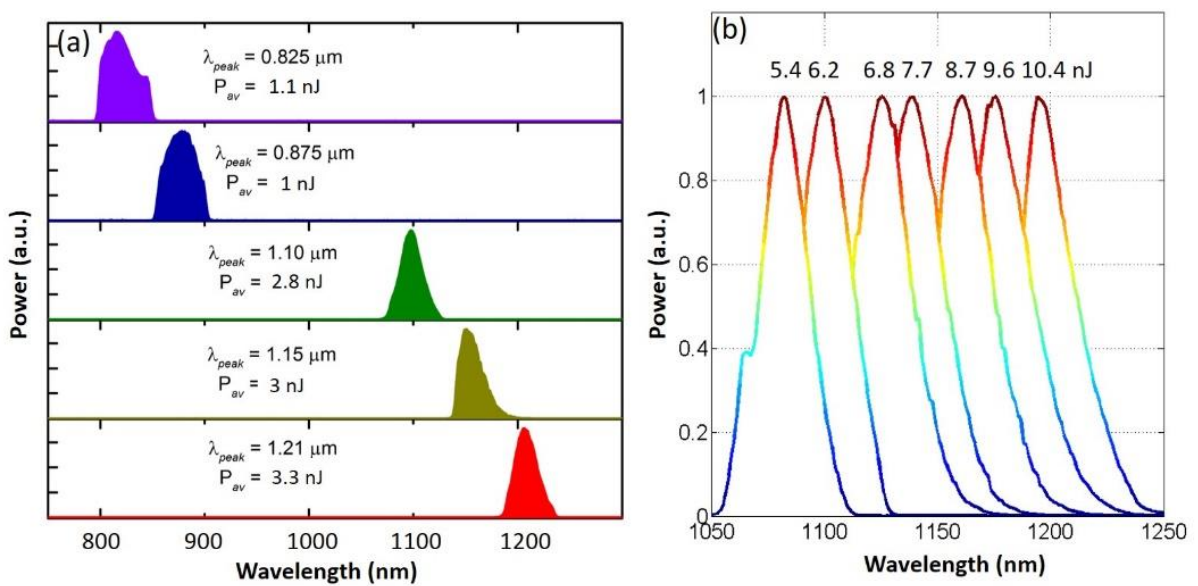

Figure 2. Filtered rightmost/leftmost spectra from SPM-enabled femtosecond fiber sources using (a) 20-mm PCF NL-1050-ZERO-2 and (b) 70-mm LMA-8 by varying the input pulse energy.

Higher pulse energy (e.g., >10-nJ level) is required to excite higher order nonlinear processes such as third harmonic generation or three photon fluorescence, typically applied in the wavelength regime above $1100 \mathrm{~nm}$ [13]. In addition to managing nonlinearity using fibers with different MFDs, fiber length is another degree of freedom to further scale up the pulse energy. Although much more power needs to be coupled into a shorter fiber to shift the resulting rightmost spectral lobe at a certain wavelength, the filtered spectrum from a shorter fiber result in higher pulse energy. This constitutes another powerful energy-scaling means-using shorter fibers for SPMenabled spectral broadening. For example, the filtered spectrum at $1100 \mathrm{~nm}$ with pulse energy as high as $22 \mathrm{~nJ}$ can be obtained by using $20 \mathrm{~mm}$ fiber LMA 8. Using a more powerful fiber laser source can increase the farthestreaching wavelength. By simulating a 250-nJ, 190-fs pulse propagating inside 2-cm long HI-1060 fiber, the rightmost spectral lobe is well isolated and continuously red-shifts along the fiber propagation. At the output of the 2-cm fiber, the filtered rightmost spectral lobe can be tuned to around $1310 \mathrm{~nm}$, close to the GFP three-photonexcitation wavelength, with a high $37 \mathrm{~nJ}$ pulse energy.

\section{EX VIVO SKIN BIOPSY BASED ON THE FIBER SOURCE}
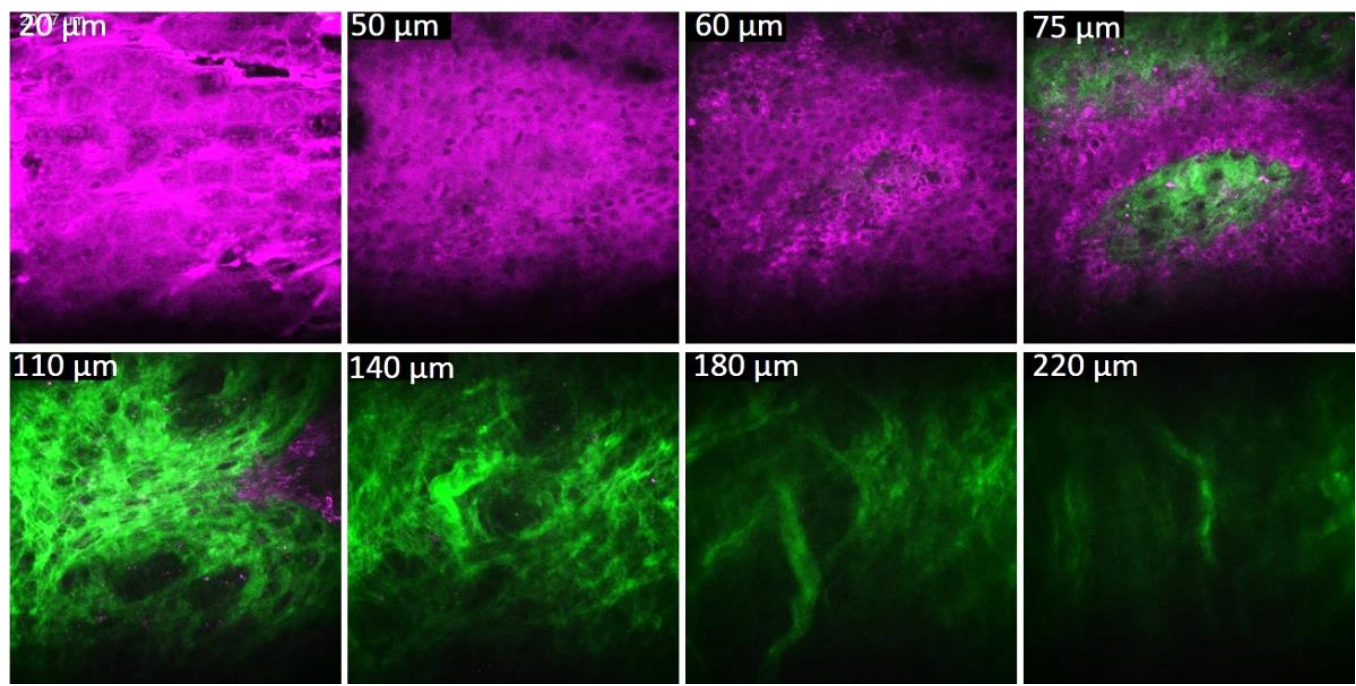

Figure 3. Horizontal-sectioned epi-SHG/THG images of ex vivo human skin at the notified imaging depth, excited by the filtered SPM-enabled source at $1200 \mathrm{~nm}$. Cell morphology from the stratum corneum to the stratum basale in epidermis can be obtained from the THG contrast. Combined with the epi-SHG modality, depth-dependent collagenous distribution in the dermis observed through epi-SHG. Magenta: THG, Green: SHG. Imaging size: $200 \mu \mathrm{m} \times 200 \mu \mathrm{m}$.

We further demonstrated the applicability of our tunable fiber source by performing ex vivo human skin observation using optical harmonics. Unlike fluorescence microscopy, optical harmonics provide naturally 
endogenous contrasts; their coherent nature offers additional insights to visualize the tissue morphology and optical structure [2]. Free from photodamage, second-harmonic generation (SHG) and third-harmonic generation (THG) images reveal different optical structures and provide complementary sub-cellular information of human skin at different layers [2]. With THG microscopy, the cellular morphology in epi-dermis can be distinguished, while the THG contrasts can also be found in dermis to show fibroblasts, erythrocytes, collagen fiber bundles, and elastin fibers [14]. On the other hand, collagen-sensitive SHG reveals the diverse collagenous structures/distribution in dermis. Figure 3 shows the combined SHG/THG images (SHG in green and THG in magenta) of ex vivo human skin from stratum corneum to the reticular dermis. We employed fiber LMA-8 to generate the filtered spectral lobe at $1200 \mathrm{~nm}$ with the illumination pulse energy of $<2 \mathrm{~nJ}$ after the objective lens to avoid sample damage. The excited harmonic signals were epi-collected by the same objective lens and separated by a dichroic mirror. The cell morphology via THG contrast in epidermis and the collagen distribution via SHG contrast in dermis can be clearly distinguished in the 10 -frame-averaged video-rate $(30 \mathrm{~Hz})$ images. $>220 \mu \mathrm{m}$ of imaging penetration depth from the top of the stratum corneum can be obtained. Due to the energy scalability and wavelength tunability of the demonstrated fiber-source, deeper penetration depth is possible by increasing the pulse energy and optimizing the excitation wavelength.

\section{CONCLUSIONS}

We have demonstrated a versatile SPM-enabled method to generate ultrafast fiber laser source for multiphoton microscopy. The method can be applied to generate $>1 \mathrm{~nJ}$ femtosecond driving sources for multiphoton microscopy, widely tunable between $825-1210 \mathrm{~nm}$, without the use of expensive and complicated Ti:sapphirebased systems. More pulse energy, up to sub-100nJ level, can be achieved by further shortening the fiber and increasing the coupled power. We applied such a high-energy source to a preliminary feasibility test by performing ex vivo human skin observation using THG/SHG contrasts. The horizontal histology section from stratum corneum to the reticular dermis can be obtained with $>220 \mu \mathrm{m}$ penetration depth. Since all the free-space components in our source demonstration can be replaced by fiber or fiber-pigtailed devices, sub-100-nJ-level femtosecond sources in an all-fiber format are possible even for three-photon fluorescence imaging.

\section{ACKNOWLEDGEMENTS}

The authors acknowledge financial support by the excellence cluster "The Hamburg Centre for Ultrafast Imaging Structure, Dynamics and Control of Matter at the Atomic scale" of the Deutsche Forschungsgemeinschaft.

\section{REFERENCES}

[1] W. R. Zipfel, R. M. Williams, W. W. Webb: Nonlinear magic: multiphoton microscopy in the biosciences," Nature Biotechnology, vol. 21, pp. 1369-1377, Nov. 2003.

[2] S.-Y. Chen, H.-Y. Wu, and C.-K. Sun: In vivo harmonic generation biopsy of human skin, J. Biomed. Opt., vol. 14, 060505, Nov. 2009.

[3] M. J. Koehler, K. König, P. Elsner et al.: In vivo assessment of human skin aging by multiphoton laser scanning tomography, Opt. lett., vol. 31, pp. 2879-2881, Oct. 2006.

[4] N. G. Horton, K. Wang, D. Kobat et al.: In Vivo three-photon microscopy of subcortical structures within an intact mouse brain, Nature Photon., vol. 7, pp. 205-209, Jan 2013.

[5] J. Huisken, J. Swoger, F. Del Bene et al.: Optical sectioning deep inside live embryos by selective plane illumination microscopy, Science, vol. 305, pp. 1007-1009, Aug. 2004.

[6] T. Wilson: Confocal microscopy, Academic Press, vol. 426, pp. 1-64, 1990.

[7] M. Erkintalo, C. Aguergaray, A. Runge et al.: Environmentally stable all-PM all-fiber giant chirp oscillator, Opt. Express, vol. 20, pp. 22669-22674, Sep. 2012.

[8] J. Szczepanek, T. M. Kardas, M. Michalska et al:: Simple all-PM-fiber laser mode-locked with a nonlinear loop mirror, Opt. Lett., vol. 40, pp. 3500-3503, Aug. 2015.

[9] X. Liu, C. Xu, W. H. Knox et al.: Soliton self-frequency shift in a short tapered air-silica microstructure fiber, Opt. Lett., vol. 26, pp. 358-360, Mar. 2001.

[10] M. E. Pedersen, J. Cheng, K. Charan et al.: Higher-order-mode fiber optimized for energetic soliton propagation, Opt. Lett. vol. 37, 3459-3461, Aug. 2012.

[11] W. Liu, C. Li, Z. G. Zhang et al: Self-phase modulation enabled, wavelength tunable ultrafast fiber laser sources: an energy scalable approach, Opt. Express, vol. 24, pp. 15328-15340, Jul. 2016.

[12] W. Liu, S.-H. Chia, H.-Y. Chung et al.: Energetic ultrafast fiber laser sources tunable in 1030-1215 nm for deep tissue multi-photon microscopy, Opt. Express, vol. 25, pp. 6822-6831, Mar. 2017.

[13] J. Y. Huang, L. Z. Guo, J. Z. Wang et al.: Fiber-based 1150-nm femtosecond laser source for the minimally invasive harmonic generation microscopy, J. biomed. Opt., vol. 22, 036008, Mar. 2017.

[14] C.-H. Yu, S.-P. Tai, C.-T. Kung et al.: In vivo and ex vivo imaging of intra-tissue elastic fibers using thirdharmonic-generation microscopy, Opt. Express, vol. 15, pp. 11167-11177, Sep. 2007. 